\title{
Structure-Corrosion Inhibition Performance Relationship: Application to Some Natural Free Acids and Antioxidants
}

\author{
Nigri Soraya ${ }^{a,{ }^{*},}$ Djemil Rayenne ${ }^{b}$, \\ Messaoudi Boulanouar ${ }^{c}$ and Oumeddour $\operatorname{Rabah}^{a}$ \\ ${ }^{a}$ Laboratory of Industrial Analysis and Materials Engineering. University May 8, 1945, \\ P.O. Box 401, Guelma, 24000, Algeria \\ ${ }^{b}$ Laboratory of Computational Chemistry and Nanostructures. University May 8, 1945, \\ P.O. Box 401, Guelma, 24000, Algeria \\ ${ }^{c}$ Laboratory of Applied Thermodynamics and Molecular Modelling, Department of Chemistry, \\ University of Tlemcen, P.O. Box 119, Tlemcen, 13000, Algeria
}

Received December 22, 2016; accepted June 30, 2017

\begin{abstract}
The inhibition of mild steel corrosion in a $1 \mathrm{M} \mathrm{HCl}$ solution by some natural free acids and antioxidants has been investigated by weight loss measurement, potentiodynamic polarization and DFT calculations. The experimental results have shown that these compounds exhibited a good corrosion inhibition. The inhibition efficiency increased with the inhibitor concentration. The adsorption of the inhibitor molecules onto the metal surface was found to respond to Langmuir adsorption isotherm for ascorbic, oleic and stearic acids, and to Temkin adsorption isotherm for palmitic acid. Tafel plot analysis revealed that these compounds acted as mixed type inhibitors, with more polarized cathodic than anodic curves. Regarding quantum chemical calculations, parameters such as energies of highest occupied molecular orbital and lowest unoccupied molecular orbital, energy gap, dipole moment, electronegativity, global hardness, softness, global electrophilicity, fraction of transferred electrons, $\Delta \mathrm{E}$ Backdonation, Fukui and local softness indices have been performed on the tested inhibitors to investigate their structural and electronic properties, in order to provide an adsorption mechanism, and reveal the reactivity and selectivity of the molecules' centers. The experimental results were in good agreement with theoretical results. The results for the natural acids were used to predict the linoleic acid inhibition efficiency.
\end{abstract}

Keywords: corrosion inhibition; free acid; electrochemical measurements; weight loss; quantum chemical calculations.

\section{Introduction}

Corrosion is a complex and multifaceted phenomenon that adversely affects and causes deterioration in metals through oxidation. Huge losses in the metal

\footnotetext{
* Corresponding author. E-mail address: nigri_@yahoo.fr
} 
industry can be attributed to metal corrosion. Several methods can be employed to minimize the metallic corrosion rate. These methods vary depending on different factors, such as metal type, environment, corrosion agent species, and others, to ensure effective corrosion prevention that can last for a long time [1]. Among the several corrosion control strategies, the use of chemical inhibitors has proved to be the most effective and economic corrosion prevention method [2]. However, most of the available inhibitors are toxic compounds [3], and do not completely fulfill the requirements imposed by environmental protection standards. The new generation of environmental regulations requires the replacement of toxic chemicals by new environmentally friendly inhibitors [4]. Nowadays, natural products from plant extracts, and substances from other renewable sources are nontoxic, biodegradable, relatively cheap and completely soluble in aqueous media. Those natural products have been reported in several studies as green effective metal corrosion inhibitors in different aggressive environments [5].The green inhibitors can be used in the form of extracts [6-9], essential oils [10-12] or pure compounds [13-15].

Organic inhibitors can adsorb onto the metal/solution interface via three distinct mechanisms: $\pi$-bond orbital adsorption, electrostatic adsorption and chemisorption [16].

These organic corrosion inhibitors efficiency is related to the presence of polar functional groups with nitrogen, sulfur and oxygen in the molecule, and of heterocyclic compounds with polar functional groups and conjugated double bonds [17-19]. The polar function is generally regarded as the reaction center for the adsorption process occurrence [1].

A high number of works has reported theoretical studies, in order to correlate structural and electronic parameters to the inhibition efficiency, and also to explain the corrosion inhibition mechanism [20-22]. These results have been used to accurately interpret many experimental phenomena, design new inhibitors and predict corrosion efficiency for related structures.

The aim of this study is to explore the use of some free acids and natural antioxidants, using vegetable oils or biomass extracts, rich on these fatty acids, as future green corrosion inhibitors. The corrosion inhibition efficiency has been experimentally evaluated for a mild steel surface in a $\mathrm{HCl}$ solution, using weight loss and potentiodynamic polarization techniques, as well as quantum chemical calculations.

Quantum chemical calculations have been used to study a reactive mechanism. They are also an effective tool in the analysis and elucidation of many experimental observations, and, in this work, they have proved to be a very powerful tool for studying the corrosion inhibition mechanism. Parameters, such as energies of highest occupied molecular orbital ( $\left.\mathrm{E}_{\text {Номо }}\right)$ and lowest unoccupied molecular orbital ( $\left.\mathrm{E}_{\mathrm{LUMO}}\right)$, energy gap $(\Delta \mathrm{E})$, dipole moment $(\mu)$, electronegativity $(\chi)$, global hardness $(\eta)$, softness $(\sigma)$, global electrophilicity $(\omega)$, fraction of electrons transferred $(\Delta \mathrm{N})$ and $\Delta \mathrm{E}$ back-donation have been calculated and used to analyze the molecular electronic structures of tested inhibitors, as well as to probe the mechanism of metal-inhibitor interactions. The local reactivity has been analyzed by means of Fukui functions, since they indicate the reactive 
region, in the form of the nucleophilic and electrophilic behavior of each atom in the inhibitor's molecules.

\section{Experimental}

The mild steel used for this study was provided in a rectangular form of $1 \times 2$ $\mathrm{cm}^{2}$ surface, with the following composition (wt. \%): C 0.07, Si 0.039, Mn 0.27, $\mathrm{P}$ 0.08, S 0.08, Al 0.031, Cu 0.042, Cr 0.009, Ni 0.014 and Fe balance. Before use, these specimens were polished with grade 600, 1200 emery paper, degreased in absolute ethanol, and then rinsed with bidistilled water.

The aggressive solution, $1 \mathrm{M} \mathrm{HCl}$, was prepared by dilution of analytical grade $37 \%$ hydrochloric acid, with bidistilled water.

The tested inhibitors in this study are oleic (OA), palmitic (PA), stearic (SA) and ascorbic (AA) acids.

Inhibitor solutions were prepared by dissolving desired amounts in $1 \mathrm{M}$ solutions, to yield different concentrations in each corrosive medium.

Weight loss measurements were recorded in a thermostated water bath in a $1 \mathrm{M}$ $\mathrm{HCl}$ solution, with and without the addition of different inhibitors concentrations for various intervals of time. They were then taken out and washed with bidistilled water and ethanol. The corrosion product on the metal surface was mechanically removed by rubbing it with a brush. The weight of each specimen was measured before and after testing in the aggressive medium, with an analytical balance (precision $\pm 0.1 \mathrm{mg}$ ).

Potentiodynamic polarization curves were recorded by the use of a 273A EG\&G PAR potentiostat combined with "Power suite software"; the scan rate was 50 $\mathrm{mV} / \mathrm{s}$. The potential ranged from -800 to $+0 \mathrm{mV} / \mathrm{Ag} / \mathrm{AgCl}$. Electrochemical measurements were performed with a conventional three-electrode electrochemical cell (with thermostat), consisting of platinum as auxiliary electrode, silver/silver chloride $(\mathrm{Ag} / \mathrm{AgCl})$ as reference electrode, and mild steel as working electrode.

The theoretical study of corrosion inhibitors was done by using the Density Functional Theory (DFT) with the B3LYP /6-31G(d) [23] method implemented in Gaussian 09 program package [24].

\section{Results and discussion}

The inhibitors were tested at different concentrations for the optimum time of 3 h. The corrosion rates (CR), inhibition efficiencies (EI) and surface coverage $(\theta)$, in the inhibitor presence, were calculated. It is clear that the corrosion rate markedly decreased, and that the inhibition efficiency increased with an increasing inhibitor concentration. The best results in terms of inhibition efficiencies are given in Fig. 1.

At concentrations of $8 \times 10^{-4} \mathrm{M}, 0.6 \% \mathrm{~V} / \mathrm{V}, 10^{-3} \mathrm{M}$ and $4 \times 10^{-4} \mathrm{M}$, the maximum obtained EI\% were $90 \%, 86 \%, 75 \%, 74 \%$ and $86 \%$ for, respectively, AA, OA, SA, PA and LA. 
This allows concluding that the tested inhibitors have promising corrosion inhibitor properties for mild steel in $\mathrm{HCl}$.

According to the inhibitors concentrations, the $\boldsymbol{\theta}$ variation indicates the adsorption isotherm that describes the system.

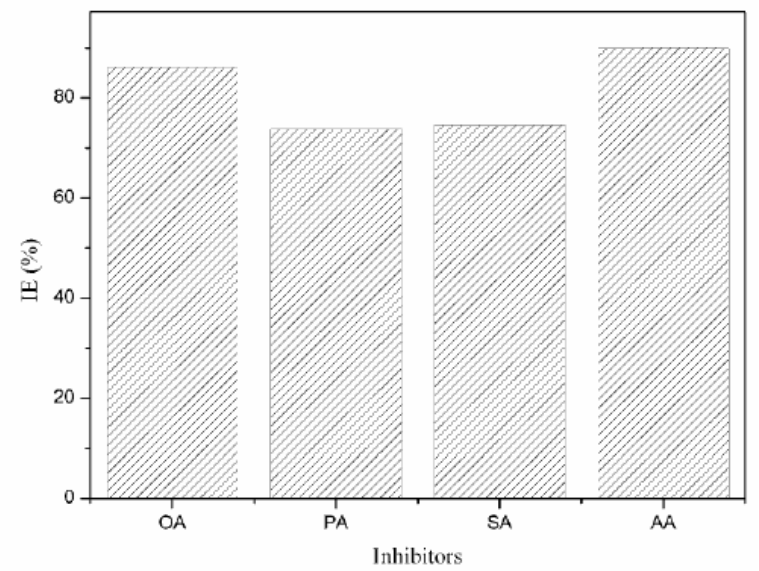

Figure 1. Inhibition efficiency of different inhibitors at optimum concentrations.

To clarify the nature of this adsorption, and the type of interaction between the inhibitor molecules and the mild steel surface, Langmuir, Frumkin and Temkin isotherms were applied. They were also used to fit into the degree of surface coverage $(\boldsymbol{\theta})$ values at different inhibitors concentrations. The data were graphically tested to find a suitable adsorption isotherm. The correlation coefficient, R, was used as a criterion to select the suitable adsorption isotherm.

The fit of Langmuir isotherm offers excellent models, with $\mathrm{R}=0.94,0.99$ and 0.99, respectively, for AA, OA and SA.

According to Langmuir adsorption isotherm:

$$
\frac{c}{\theta}=\frac{1}{k_{a d s}}+c
$$

where $\mathrm{C}$ represents the inhibitor concentration, $\boldsymbol{\theta}$ is the degree of surface coverage on the metal surface, and $\mathrm{K}_{\mathrm{ads}}$ is the equilibrium constant for the adsorption-desorption process.

Fig. 2 ( $\mathrm{a}, \mathrm{b}, \mathrm{c}$ ) shows that the plot of $\mathrm{C} / \boldsymbol{\theta}$ against $\mathrm{C}$ is linear for, respectively, AA, $\mathrm{OA}$ and SA. The linear plots slopes were greater than unity. This can be attributed to the interactions between adsorbate species on the metal surface, as well as to changes in the adsorption heat, with an increasing surface coverage; factors which were not taken into consideration in deriving the isotherm.

PA was found to obey Temkin adsorption isotherm, with $\mathrm{R}=0.99$.

The degree surface coverage values for PA were fitted into the Temkin adsorption isotherm model, which has the form

$$
\exp (-2 a \theta)=K_{a d s} C
$$

where " $a$ " is the molecules interaction parameter.

The degree surface coverage plot, as a function of the inhibitors concentration logarithms, is shown in Figure 2 (d) (Temkin adsorption isotherm). From the 
plot, straight lines were obtained with a positive slope and a negative parameter of molecules interaction, showing that repulsion exists in the adsorption layer.
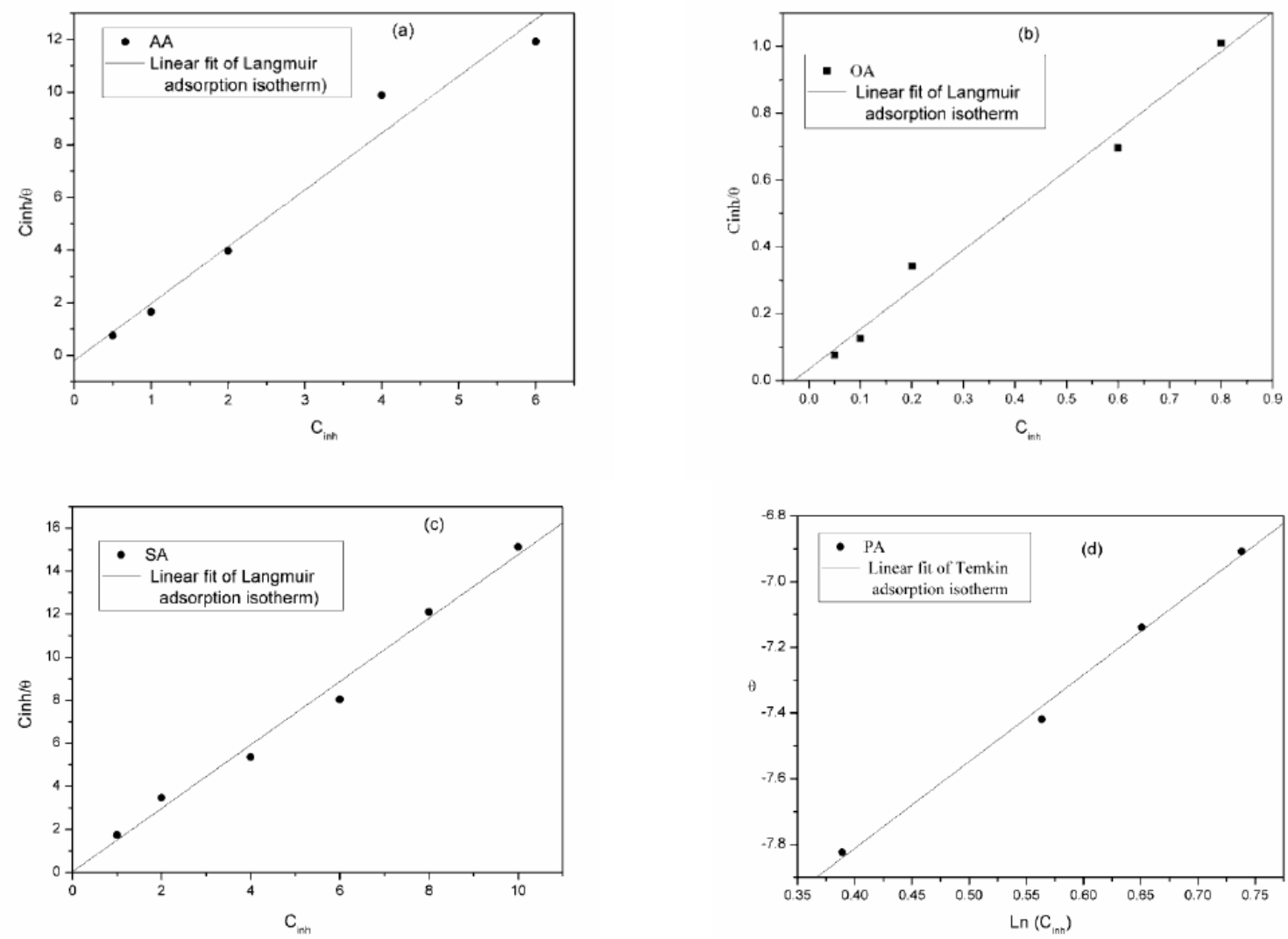

Figure 2. Langmuir adsorption model for (a) AA, (b) OA and (c) SA; and (d) Temkin adsorption isotherm for PA.

Polarization measurements were carried out, in order to gain knowledge regarding the kinetics of cathodic and anodic reactions, and to determine how the inhibitory effect acted. Polarization curves obtained for mild steel, in the absence and presence of inhibitors at optimum concentration, are shown in Fig. 3.

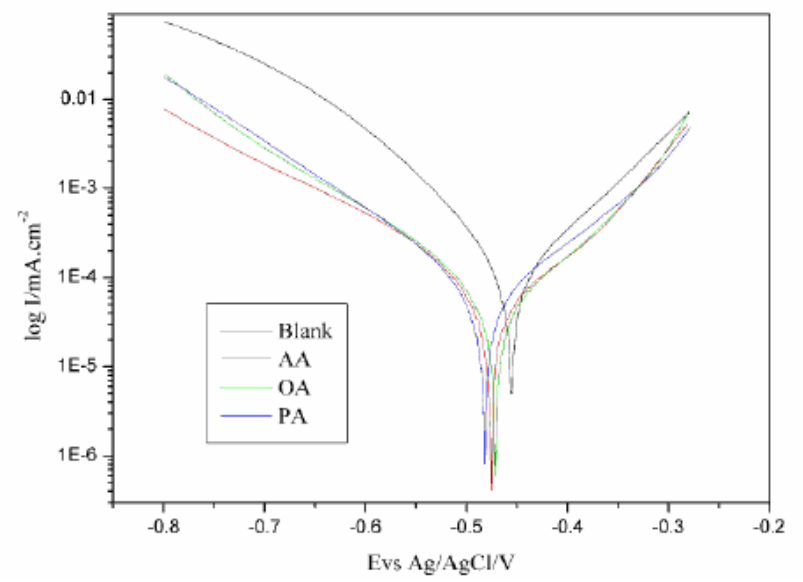

Figure 3. Potentiodynamic polarization curves for mild steel in $1 \mathrm{M} \mathrm{HCl}$, without and with tested inhibitors. 
Inspection of Tafel curves reveals that corrosion current densities are significantly reduced in the inhibitors presence. The displacement in Ecorr is < $85 \mathrm{mV}$; with respect to the blank acid solution's Ecorr, AA, OA, PA and SA can be considered as mixed type inhibitors [25]. It is also observed that the tested inhibitors addition retards both cathodic and anodic reactions; however, cathodic reactions are comparatively more affected than anodic ones, suggesting that investigated inhibitors are mixed type inhibitors, and predominantly act as cathodic inhibitors.
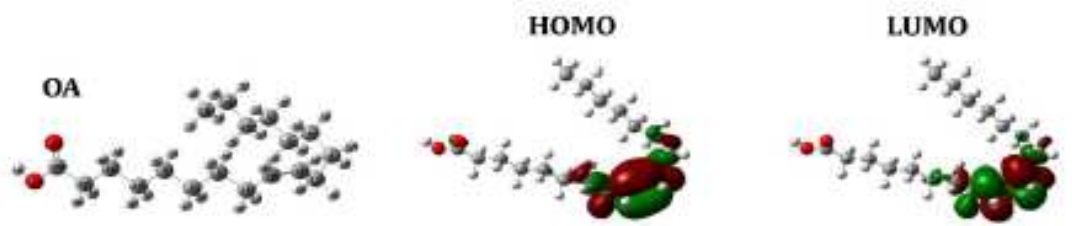

PA

номо

LUMO
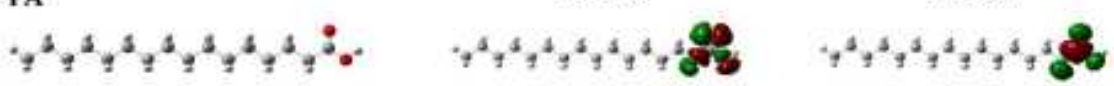

SA

номо

LUMO

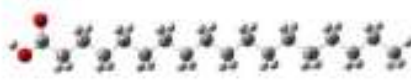
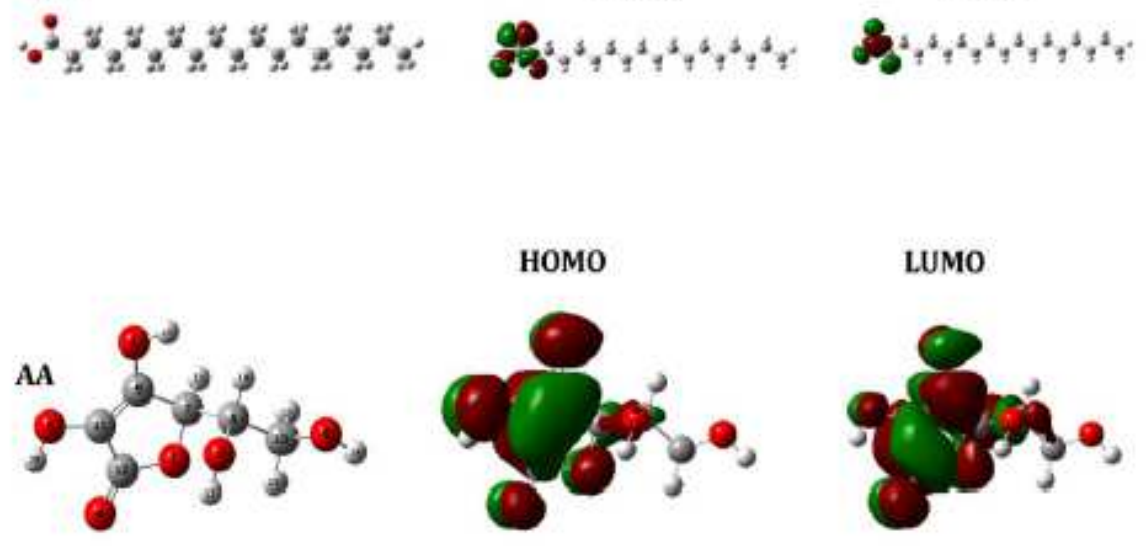

LUMO

номо
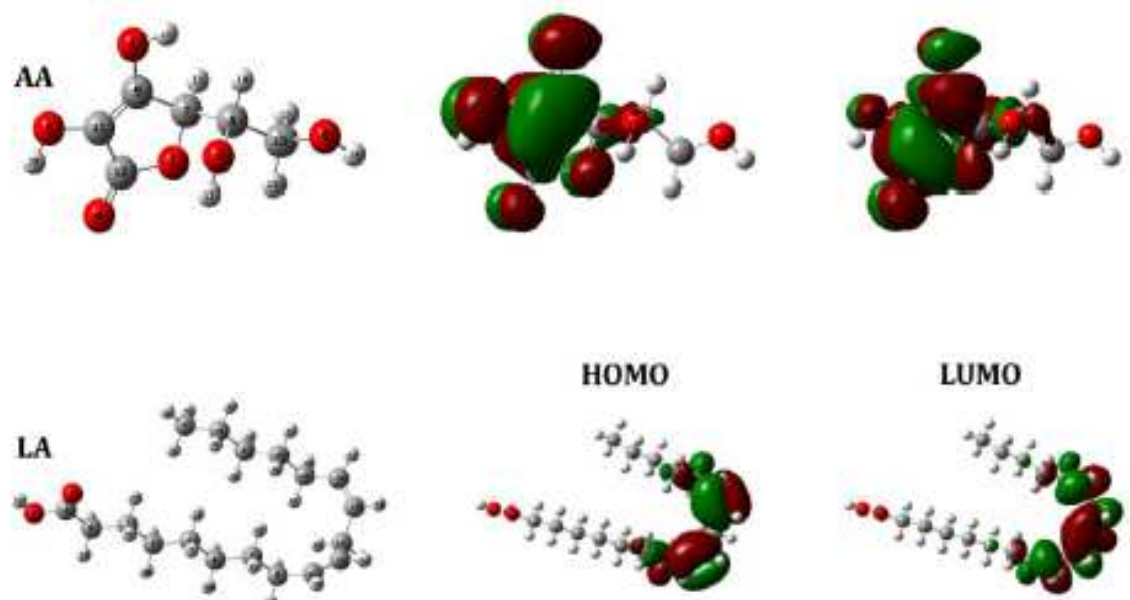

Figure 4. The optimized structure, HOMO and LUMO distribution, for OA, PA, SA, AA and LA [atom legend: white $=\mathrm{H}$; gray $=\mathrm{C}$; red $=\mathrm{O}$ ]. 
Hydrogen evolution reactions were significantly influenced by the inhibitors presence in the corrosive medium. The corrosion rate decrease is more pronounced in the AA presence. This is attributed to the inhibitor adsorption on active metal sites, present on the mild steel surface, which is the primary step in achieving-inhibition in the $\mathrm{HCl}$ solution.

The obtained results from Tafel polarization showed good agreement with the weight loss measurements ones.

Quantum chemical approach, using the density functional theory (DFT), was used in order to get a better understanding of the relationship between the tested inhibitors inhibition efficiency and molecular structure. The calculated quantum chemical parameters include: the energies of highest occupied molecular orbital $\left(\mathrm{E}_{\text {Hомо }}\right)$ and lowest unoccupied molecular orbital ( $\left.\mathrm{E}_{\mathrm{LUMO}}\right)$, energy gap $(\Delta \mathrm{E})$, dipole moment $(\mu)$, electronegativity $(\chi)$, global hardness $(\eta)$, softness $(\sigma)$, global electrophilicity $(\omega)$, fraction of transferred electrons $(\Delta \mathrm{N})$ and $\Delta \mathrm{E}$ Back-donation. The local reactivity has been analyzed through Fukui and local softness indices, to compare possible sites for nucleophilic and electrophilic attacks.

The optimized structure, EHOMO and ELUMO, of all inhibitors is shown in Fig. 4.

Table 1 lists quantum chemical parameters calculated for geometrically optimized molecules.

Table 1. Quantum-chemical properties calculated for the most stable conformations of OA, PA, SA, AA and LA (linoleic acid).

\begin{tabular}{cccccc}
\hline Properties & OA & PA & SA & AA & LA \\
\hline Eномо $(\mathbf{e v})$ & -6.3825 & -7.4345 & -7.43402 & -6.2277 & -6.3406 \\
$\mathbf{E}_{\mathbf{L U M O}}(\mathbf{e v})$ & 0.3303 & 0.3424 & 0.3427 & -0.8508 & 0.3272 \\
$\mathbf{\Delta E}(\mathbf{e v})$ & 6.7126 & 7.7769 & 7.7767 & 5.3768 & 6.6678 \\
$\boldsymbol{\mu}(\mathbf{D e b y e})$ & 1.4320 & 1.3647 & 1.3644 & 3.6700 & 1.4841 \\
$\mathbf{I}=-\mathbf{E}$ HOMо & 6.3825 & 7.4345 & 7.43402 & 6.2277 & 6.3406 \\
$\mathbf{A}=-\mathbf{E}_{\mathbf{L U M O}}$ & -0.3303 & -0.3424 & -0.3427 & 0.8508 & -0.3272 \\
$\chi=(\mathbf{I}+\mathbf{2}) / \mathbf{2}$ & 3.0261 & 3.54603 & 3.54566 & 3.53925 & 3.0067 \\
$\eta=(\mathbf{I}-\mathbf{2}) / \mathbf{2}$ & 3.3564 & 3.8885 & 3.8884 & 2.6885 & 3.3339 \\
$\boldsymbol{\sigma}=\mathbf{1} / \eta$ & 0.2973 & 0.2572 & 0.2572 & 0.3720 & 0.2999 \\
$\boldsymbol{\omega}=\mu^{2} /(\mathbf{2} \eta)$ & 0.3055 & 0.2395 & 0.2394 & 2.5050 & 0.3303 \\
$\Delta \mathbf{E}_{\text {back donation }}=-\eta / \mathbf{4}$ & -0.8391 & -0.9721 & -0.9721 & -0.6721 & -0.8335 \\
$\Delta \mathbf{N}=\left(\chi_{\mathbf{F e}}-\chi_{\text {inh }}\right) /\left(2\left(\eta_{\mathrm{Fe}}-\eta_{\text {inh }}\right)\right)$ & 0.21659 & 0.1201 & 0.1201 & 0.1750 & 0.5989 \\
\hline
\end{tabular}

In this study, a good correlation has been obtained between the corrosion

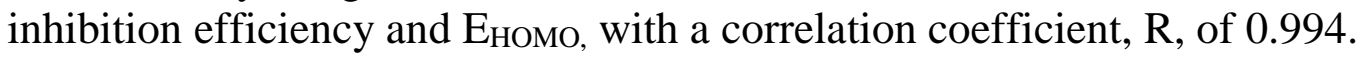

The obtained intercept and the slope are, respectively, 0.000 and 1.000. This result indicates that the inhibitors adsorption onto the metal surface can occur on the basis of donor-acceptor interactions between the inhibitors' $\pi$ electrons and the vacant d-orbitals of the metal surface atoms. These interactions are often 
associated to HOMO's energy. The higher are Eномо's values, greater is the inhibition efficiency. This can be explained by the influence applied on the transport through the adsorbed layer [26].

Organic compounds are well-known to be excellent corrosion inhibitors. They do not only offer electrons to the metal's unoccupied orbital, but also accept free electrons from it [27]. The ability of the inhibitor's molecule to accept electrons from the metal's d-orbital is often associated to LUMO's energy (ELUMO). The negative value $(-0.733)$ of the correlation coefficient, $R$, confirmed the observation that the lower is ELUMO, the easier is the acceptance of electrons from metal surface atoms.

The energy gap ( $\Delta E=E_{\text {Homo }}-E_{\text {Lumo }}$ ) is found to be an important factor used as a descriptor of molecules reactivity. It indicates the adsorption capacity onto the metallic surface [28]. It has been shown that the smaller is the gap HOMOLUMO, the more reactive is an electronic system. Therefore, inhibitors with a small energy gap are the most effective for metal corrosion inhibition. From the results of Table 1, the high AA inhibition efficiency can be attributed to the highest $\mathrm{E}_{\mathrm{HOMO}}$ and the smallest $\Delta \mathrm{E}$ and $\mathrm{E}_{\mathrm{Lumo}}$ values.

Dipole moment is another parameter of such importance. It is a measure of a molecule's overall polarity. As the dipole moment value increases, so does the molecule's polarity. It can be seen from Table 1 that the inhibition efficiency increases with an increasing inhibitors dipole moment. This is confirmed by the correlation coefficient positive value, $R$, of 0.745 . These findings are in good agreement with those obtained by Obot et al. [29]. Actually, the dipole moment's higher value increases the adsorption strength between the inhibitor and the metal surface, which explains the highest AA inhibition efficiency. This adsorption results from the electrostatic interaction between AA molecules' charged centers and the charged metal surface.

Moreover, the ionization energy is undoubtedly a fundamental descriptor of molecules chemical reactivity; while higher ionization energy indicates higher stability and chemical inertness, a lower one indicates higher molecules reactivity [30]. The lower AA ionization energy, $6.2277 \mathrm{eV}$, clearly explains the higher inhibition efficiency of this compound.

In addition, absolute hardness, $\eta$, is defined as the second energy derivative, with respect to the electrons' number [31]. Within the framework of Koopman's theorem [32], high hardness values are related to the molecule stability, as well as to the HOMO-LUMO energy gap. The absolute softness is also an indication of high reactivity. In the present study, AA, characterized by low hardness $(2.68845 \mathrm{eV})$ and high softness $(0.37196 \mathrm{eV})$ values, with respect to the other inhibitors, is, then, less stable and more reactive against iron corrosion.

Adding to this, electrophilicity, which is a reactivity descriptor, provides a quantitative classification of a molecule global electrophilic nature, within a relative scale. It absolutely is the power of a system to 'soak up' electrons [33]. A good electrophile is characterized by a high $\omega$ value; whereas a good nucleophile is characterized by a low $\omega$ value. This reactivity index measures the system stabilization in terms of energy, when an additional electronic charge, $\Delta \mathrm{N}$, is acquired from the environment. The results presented in Table 1 show that $\omega$ is 
the highest for AA, and the smallest for SA. This means that AA has the highest capacity to accept electrons from the partially filled iron d-orbital, whereas SA has the highest capacity to donate electrons to the unfilled iron d-orbital. This index gives an idea about the tested inhibitors adsorption mechanism.

The number of transferred electrons $(\Delta N)$ was also calculated according to Pearson [34], and presented in Table 1. If $\Delta \mathrm{N}<3.6$, the inhibition efficiency increases by increasing the ability of these inhibitors to donate electrons to the metal surface [35]. On this basis, the following order was found: $\mathrm{OA}>\mathrm{AA}>\mathrm{SA}>\mathrm{PA}$.

It is important to note that $\Delta \mathrm{N}$ values strongly correlate with experimental inhibition efficiencies, with a correlation coefficient, R, of 0.989 ; also, when using quantum chemical indices, the best linear model was obtained by two variables, Eномо and $\Delta \mathrm{N}$, with a correlation coefficient, $\mathrm{R}$, of 0.999 , and a standard deviation (SD) of 0.533 .

Fig. 5 exhibits the scatter plot for the relationship between the inhibitors predicted and experimental IE (\%) values.

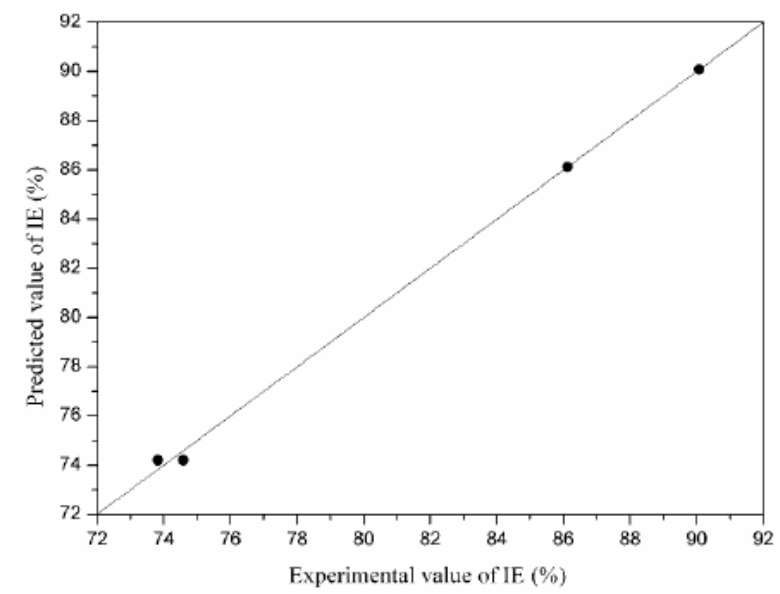

Figure 5. Correlation plot of the predicted IE (\%) value versus the experimental IE (\%) value.

As it can be seen from Fig. 5, the obtained model, using the variables $\mathrm{E}$ номо and $\Delta \mathrm{N}$, was able to give a very good correlation, with $\mathrm{R}=1.000$. The intercept and the slope of the predicted vs. experimental results for calibration data are, respectively, 0.000 and 1.000 . This confirms the model fitting power.

This model was also used to predict linoleic acid (LA) inhibition efficiency, which was $86 \%$.

On the one hand, the inhibition efficiency of the studied compounds (AA, OA, PA, SA and LA) depends on many major factors, such as the number of adsorption active centers in the molecule, and their charge density, the molecule size, mode of adsorption, and formation of a metallic complex [36]. Oxygen atoms, as well as some carbons atoms, carry negative charge centers which could offer electrons to the metal surface unoccupied orbital, forming a coordinate bond. On the other hand, the substituent effect on the mild steel inhibition is of such importance that could be rationalized using Fukui indices, $f^{+}$and $f^{-}$. While $f$ + measures the changes of density when the molecules gain electrons, and it 
corresponds to reactivity regarding a nucleophilic attack, $f$ - corresponds to reactivity regarding an electrophilic attack [37].

Table 2. Fukui indices of the selected atoms using MK and NPA population analyses at B3LYP/6-31G(d) level of theory.

\begin{tabular}{|c|c|c|c|c|c|c|c|c|}
\hline & \multicolumn{4}{|c|}{ MK } & \multicolumn{4}{|c|}{ NPA } \\
\hline & $f+$ & $f-$ & $s+$ & $s-$ & $f+$ & $f-$ & $s+$ & $s-$ \\
\hline \multicolumn{9}{|c|}{ AO } \\
\hline C16 & 0.10174 & 0.19029 & 0.41226 & 0.77104 & 0.14198 & 0.20295 & 0.57531 & 0.82236 \\
\hline C17 & 0.16102 & 0.28870 & 0.65245 & 1.16981 & 0.13221 & 0.19681 & 0.53572 & 0.79748 \\
\hline $\mathrm{C2O}$ & 0.15286 & -0.01195 & 0.61938 & -0.04841 & 0.17963 & 0.00915 & 0.72787 & 0.03708 \\
\hline 01 & 0.06013 & 0.05359 & 0.24366 & 0.21716 & 0.04706 & 0.03983 & 0.19069 & 0.16139 \\
\hline 02 & 0.12388 & 0.11816 & 0.50195 & 0.47877 & 0.11225 & 0.12389 & 0.45484 & 0.50201 \\
\hline \multicolumn{9}{|c|}{$\mathbf{P A}$} \\
\hline C18 & 0.23004 & -0.02116 & 0.80457 & -0.07399 & 0.23579 & 0.01135 & 0.82467 & 0.03970 \\
\hline 01 & 0.07871 & 0.06925 & 0.27530 & 0.24221 & 0.06039 & 0.05262 & 0.21121 & 0.18404 \\
\hline $\mathrm{O} 2$ & 0.15925 & 0.15953 & 0.55697 & 0.55795 & 0.14847 & 0.16563 & 0.51927 & 0.57929 \\
\hline \multicolumn{9}{|c|}{ SA } \\
\hline C10 & 0.20841 & -0.02092 & 0.72893 & -0.07317 & 1.58293 & 0.01083 & 5.53646 & 0.03788 \\
\hline 01 & 0.15830 & 0.14793 & 0.55369 & 0.51740 & 0.14469 & 0.15345 & 0.50607 & 0.53671 \\
\hline \multirow{2}{*}{\multicolumn{9}{|c|}{$\mathbf{A A}$}} \\
\hline & & & & & & & & \\
\hline C7 & -0.05451 & -0.02627 & -0.27577 & -0.13290 & -0.02150 & -0.02644 & -0.10876 & -0.13375 \\
\hline C8 & -0.03105 & -0.12600 & -0.15709 & -0.63741 & -0.00454 & -0.00550 & -0.02297 & -0.02782 \\
\hline C9 & 0.08053 & 0.14955 & 0.40737 & 0.75653 & 0.18051 & 0.14582 & 0.91314 & 0.73766 \\
\hline C10 & -0.05803 & 0.02033 & -0.29357 & 0.10282 & -0.00130 & -0.00849 & -0.00658 & -0.04295 \\
\hline C11 & 0.09185 & 0.15982 & 0.46462 & 0.80848 & 0.05720 & 0.14129 & 0.28936 & 0.71474 \\
\hline C12 & 0.17796 & -0.04162 & 0.90023 & -0.21052 & 0.17059 & -0.01714 & 0.86296 & -0.08671 \\
\hline 01 & 0.01741 & 0.07274 & 0.08810 & 0.36795 & 0.05168 & 0.04326 & 0.26143 & 0.21884 \\
\hline 02 & 0.05171 & 0.04840 & 0.26157 & 0.24482 & 0.01526 & 0.01294 & 0.07720 & 0.06546 \\
\hline $\mathbf{O 3}$ & 0.08673 & 0.08532 & 0.43873 & 0.43161 & 0.05545 & 0.11683 & 0.28050 & 0.59101 \\
\hline 04 & 0.03191 & 0.09269 & 0.16141 & 0.46889 & 0.01101 & 0.07066 & 0.05570 & 0.35745 \\
\hline 05 & 0.05288 & 0.14376 & 0.26753 & 0.72726 & 0.04873 & 0.16489 & 0.24651 & 0.83413 \\
\hline O6 & 0.16606 & 0.12655 & 0.84002 & 0.64018 & 0.16096 & 0.12401 & 0.81425 & 0.62733 \\
\hline \multicolumn{9}{|c|}{ LA } \\
\hline C12 & 0.09371 & 0.21023 & 0.38228 & 0.85757 & 0.09973 & 0.13982 & 0.40683 & 0.57037 \\
\hline C15 & 0.09816 & 0.08381 & 0.40043 & 0.34188 & 0.06412 & 0.10022 & 0.26156 & 0.40883 \\
\hline C17 & 0.10218 & 0.23217 & 0.41682 & 0.94709 & 0.10644 & 0.14484 & 0.43420 & 0.59085 \\
\hline C18 & 0.14048 & -0.01305 & 0.57308 & -0.05323 & 0.14720 & 0.00722 & 0.60047 & 0.02945 \\
\hline C19 & 0.09970 & 0.08347 & 0.40671 & 0.34050 & 0.06627 & 0.10285 & 0.27034 & 0.41956 \\
\hline 01 & 0.04996 & 0.04287 & 0.20381 & 0.17489 & 0.03898 & 0.03118 & 0.15901 & 0.12719 \\
\hline $\mathrm{O2}$ & 0.09734 & 0.09263 & 0.39710 & 0.37787 & 0.09154 & 0.09623 & 0.37342 & 0.39255 \\
\hline
\end{tabular}

The atomic gross electron densities were calculated using both Merz-SinghKollman (MK) and natural population (NPA) analyses. The algebraic values of Fukui indices $\left(f^{+}, f^{-}\right)$are given in Table 2 .

For nucleophilic attack, $\left(f^{+}\right)$the most reactive sites, using MK population analysis, are $\mathrm{C} 12, \mathrm{C} 17, \mathrm{C} 18, \mathrm{C} 10$ and $\mathrm{C} 18$, while those using NPA population analysis are C9, C20, C18, C10 and C18 for AA, OA, PA, SA and LA, respectively.

The Fukui function $\left(f^{-}\right)$is confirmed by the electrophilic attack at the sites C11, $\mathrm{C} 17, \mathrm{O} 2$, O1and $\mathrm{C} 17$, using $\mathrm{MK}$ population analysis, and $\mathrm{O} 5, \mathrm{C} 16, \mathrm{O} 2, \mathrm{O} 1$ and C17, using NPA population analysis, for AA, OA, PA, SA and LA, respectively. These atoms have a greater nucleophilic character, and are involved in the 
chemical reactivity of these molecules with the metal surface, which exhibits the adsorption mechanism.

The same results of $f^{+}$and $f^{-}$were obtained for both PA and SA, using the two population analyses, while for AA and OA they were different.

For OA, using MK analysis, C17 site is either a nucleophilic or electrophilic centre, and may share the same opportunity with $\mathrm{C} 16$ to be a nucleophilic center, but when talking about electrophilic centers, C20 site is more electrophilic than $\mathrm{C} 17$, to receive the nucleophilic attack. The same thing can be said about AA, where $\mathrm{O} 5$ is more nucleophilic than $\mathrm{C} 11 . \mathrm{C} 9$ and $\mathrm{C} 12$ have basically the same environment using NPA: for C9 is 0.18051 and for $\mathrm{C} 12$ is 0.17059 , while with $\mathrm{MK}$, for C9 is 0.08053 and for $\mathrm{C} 12$ is 0.11780 . Therefore, NPA analysis is more reliable and gives more precise and consistent values with respect to MK.

\section{Conclusion}

The inhibition effect of ascorbic acid and some free acids, such as oleic, palmitic and stearic acids, on the corrosion behavior of mild steel in a $1 \mathrm{M} \mathrm{HCl}$ solution, was studied by weight loss, electrochemical methods and quantum chemical calculations.

The inhibition efficiency of these acids on the mild steel corrosion in $1 \mathrm{M} \mathrm{HCl}$ increased with an increasing inhibitor concentration.

The potentiodynamic polarization curves indicate that these inhibitors act as a mixed type inhibitor, with more polarized cathodic than anodic curves.

The adsorption of AA, OA and SA on the metal surface followed Langmuir adsorption isotherm, however PA was found to obey Temkin adsorption isotherm.

The calculations of the tested inhibitors reactivity indices, such as the localization of frontier molecular orbitals, $E_{\text {HOMO, }} E_{\text {LUMO }}$, energy gap $(\Delta E)$, dipole moment $(\mu)$, hardness $(\eta)$, softness $(\sigma)$, fractions of transferred electrons $(\Delta N)$, electrophilicity index $(\omega)$, and charge distributions, together with local reactivity, by means of Fukui indices, are an accurate tool for interpreting the electron transfer mechanism between inhibitors' molecules and the iron surface. The obtained experimental results were in good agreement with the theoretical results.

\section{Acknowledgements}

The research is supported by the General directorate for scientific research and development (DG-RSDT) of Algeria. The authors gratefully acknowledge Prof. Affoune Abed Mohamed, who kindly allowed us to use the potentiostat, and Dr. Nacef Mouna, for her presence during the electrochemical measurements.

\section{References}

1. Fontana MG, Staehle KW. Advances in corrosion science and technology. vol 1. New York: Plenum Press; 1970.

2. Sastri VS. Green corrosion inhibitors, theory and practice. Hoboken: John Wiley \& Sons, 1998.

3. Lake DL. Corros Prevent Contr. 1988;35:113-115. 
4. Oguzie EE. Corros Sci. 2008;50:2993-2993.

5. Boumhara K, Tabyaoui M, Jama C, et al. J Ind Eng Chem. 2015;29:146-155.

6. Anupama KK, Ramya K, Joseph A. J Mol Liq. 2016;216:146-155.

7. Satapathy AK, Gunasekaran G, Sahoo SC, et al. Corros Sci. 2009;51:28482856.

8. Obi-Egbedi NO, Obot IB, Umoren SA. Arab J Chem. 2012;5:361-373.

9. Oguzie EE. Pigm Resin Technol. 2005;34:321-326.

10. Bouyanzer A, Majidi L, Hammouti B. Phys Chem News. 2007;37:70-74.

11. Bammou L, Mihit M, Salghi R, et al. Int J Electrochem Sci. 2011;6:14541467.

12. Hmamou DB, Salghi R, Zarrouk $A$, et al. Int $J$ Electrochem Sci. 2012;7:6234-6246.

13. Chidiebere MA, Oguzie EE, Liu L, et al. J Ind Eng Chem. 2015;26:182-192.

14. Obot IB, Egbedi NO. Port Electrochim Acta. 2009;27:517-524.

15. Sekine I, Nakahata Y, Tanabe H. Corros Sci. 1988;28:987-1001.

16. Gece G, Semra B. Corros Sci. 2010;52:3435-3443.

17. Kumar SR, Danaee I, Avei MR, et al. J Mol Liq. 2015;212:168-186.

18. Yadav M, Gope L, Kumari N, et al. J Mol Liq. 2016;216:78-86.

19. Migahed MA, Al-Sabagh AM, Khamis EA, et al. J Mol Liq. 2015;212:360371.

20. Youguo Y, Xiao W, Yang Z, et al. Mol Simult. 2009;39:1034-1041.

21. Umoren SA, Obot IB, Obi-Egbedi NO. J Mater Sci. 2009;44:274-279.

22. Gupta NK, Verma C, Quraishi MA, et al. J Mol Liq. 2016;215:47-57.

23. Becke AD. J Chem Phys. 1993;98:1372-1377.

24. Gaussian 09, Revision E.01. Frisch MJ, Trucks GW, Schlegel HB, et al. Gaussian, Inc., Wallingford CT; 2009.

25. Riggs O. Corrosion inhibitors. 2nd ed. Houston: C. C. Nathan, 1973.

26. Fouda AS, Abu-Elnader HM, Soliman MS. Bull Korean Chem Soc. 1986;7:97-99.

27. Zhao P, Liang Q, Li Y. Appl Surf Sci. 2005;252:1596-1607.

28. Gece G. Corros Sci. 2008;50:2981-2992.

29. Obot IB, Obi-Egbedi NO. Corros Sci. 2010;52:657-660.

30. Chakraborty T, Ghosh DC. Mol Phys. 2010;108:2081-2092.

31. Parr RG, Pearson RG. J Am Chem Soc. 1983;105:7512-7516.

32. Koopmans T. Physica. 1933;1:104-113.

33. Parr RG, Sventpaly L, Liu S. J Am Chem Soc. 1999;121:1922-1924.

34. Pearson RG. J Am Chem Soc. 1963;85:3533-3539.

35. Lukovits I, Kálmán E, Zucchi F. Corrosion. 2001;57:3-8.

36. Boussalah N, Ghalem S, El Kadiri S, et al. Res Chem Intermed. 2012;38:2009-2023.

37. Fukui K. Science. 1982;218:747-754. 